Provided for non-commercial research and education use. Not for reproduction, distribution or commercial use.

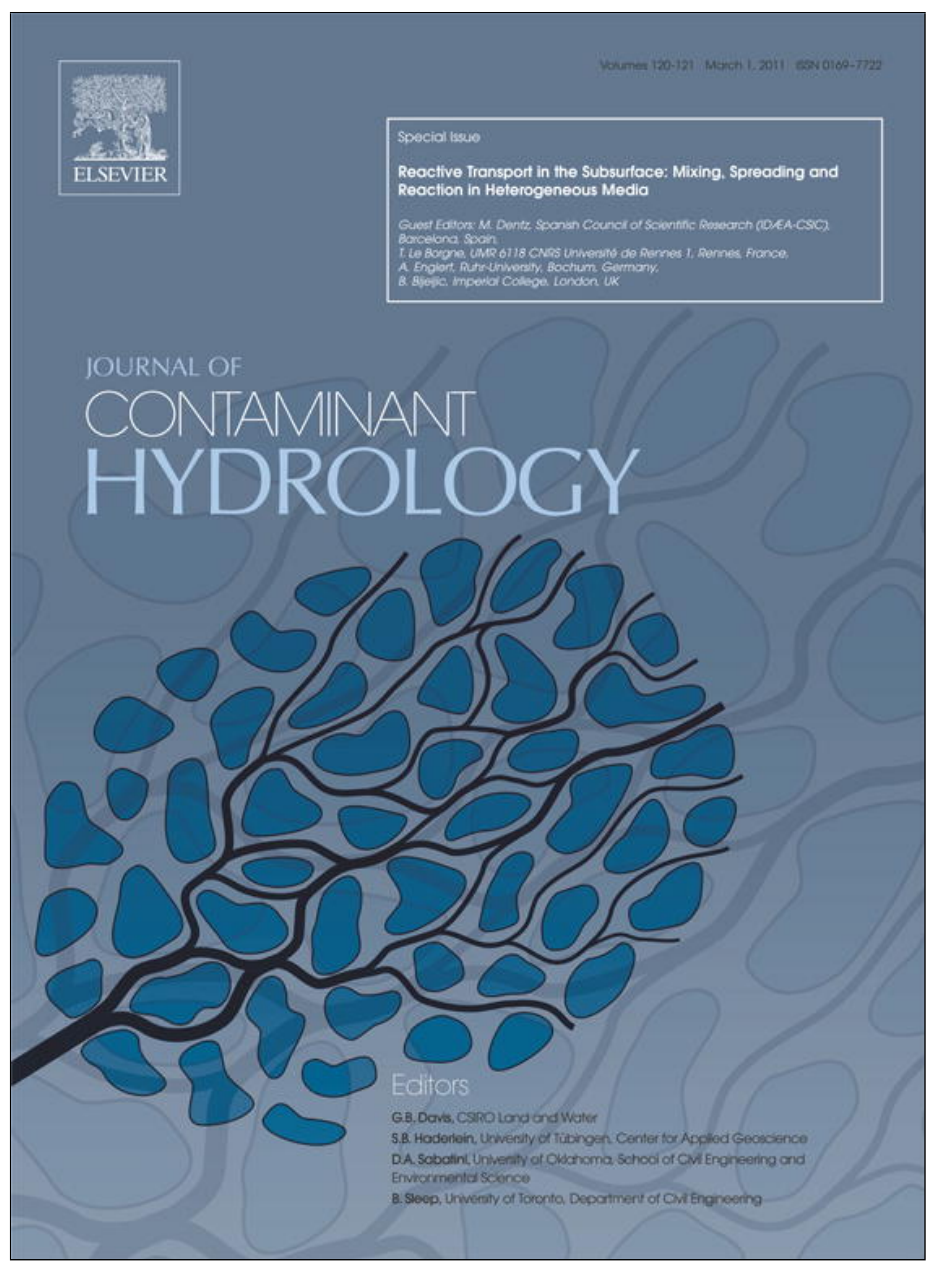

This article appeared in a journal published by Elsevier. The attached copy is furnished to the author for internal non-commercial research and education use, including for instruction at the authors institution and sharing with colleagues.

Other uses, including reproduction and distribution, or selling or licensing copies, or posting to personal, institutional or third party websites are prohibited.

In most cases authors are permitted to post their version of the article (e.g. in Word or Tex form) to their personal website or institutional repository. Authors requiring further information regarding Elsevier's archiving and manuscript policies are encouraged to visit:

http://www.elsevier.com/copyright 


\title{
NMR measurement of hydrodynamic dispersion in porous media subject to biofilm mediated precipitation reactions
}

\author{
Einar O. Fridjonsson a , Joseph D. Seymour a,b,*, Logan N. Schultz ${ }^{\text {a,b }}$, Robin Gerlach ${ }^{\text {a,b }}$, \\ Alfred B. Cunningham ${ }^{\mathrm{b}}$, Sarah L. Codd ${ }^{\mathrm{b}, \mathrm{c}}$ \\ a Department of Chemical and Biological Engineering, Montana State University, Bozeman MT 59717-3920, USA \\ b Center for Biofilm Engineering, Montana State University, Bozeman MT 59717-3980, USA \\ c Department of Mechanical and Industrial Engineering, Montana State University, Bozeman MT 59717-3800, USA
}

\section{A R T I C L E I N F O}

\section{Article history:}

Received 4 December 2009

Received in revised form 21 July 2010

Accepted 26 July 2010

Available online 3 August 2010

\section{Keywords:}

Nuclear magnetic resonance

Precipitation reaction

Biofilm

Hydrodynamic dispersion

\begin{abstract}
A B S T R A C T
Noninvasive measurements of hydrodynamic dispersion by nuclear magnetic resonance (NMR) are made in a model porous system before and after a biologically mediated precipitation reaction. Traditional magnetic resonance imaging (MRI) was unable to detect the small scale changes in pore structure visualized during light microscopy analysis after destructive sampling of the porous medium. However, pulse gradient spin echo nuclear magnetic resonance (PGSE NMR) measurements clearly indicated a change in hydrodynamics including increased pore scale mixing. These changes were detected through time-dependent measurement of the propagator by PGSE NMR. The dynamics indicate an increased pore scale mixing which alters the preasymptotic approach to asymptotic Gaussian dynamics governed by the advection diffusion equation. The methods described here can be used in the future to directly measure the transport of solutes in biomineral-affected porous media and contribute towards reactive transport models, which take into account the influence of pore scale changes in hydrodynamics.
\end{abstract}

(c) 2010 Elsevier B.V. All rights reserved.

\section{Introduction}

Reactive transport in porous media is an important problem in understanding the spread of contaminants in the Earth's subsurface. Precipitation reaction phenomena are important in transport processes of transuranic environmental contaminant species (Kersting et al., 1999; Nagy et al., 1991), carbon sequestration (Kaszuba et al., 2005), soil and slope stabilization, dam and levee safety, erosion prevention, and subsurface barrier formation for environmental restoration technologies (Cunningham et al., 2009; Decho, 2010; DeJong et al., 2010). Precipitation and dissolution reactions during flow of fluids in porous media represent a particular level of complexity for transport modeling, due to the

\footnotetext{
* Corresponding author. Department of Chemical and Biological Engineering, Montana State University, Bozeman MT 59717-3920, USA.

E-mail address: jseymour@coe.montana.edu (J.D. Seymour).
}

evolution of the pore structure due to reaction, altering flow dynamics (Emmanuel and Berkowitz, 2005; Emmanuel and Berkowitz, 2007; Sahimi et al., 1990; Tartakovsky et al., 2007). Models for these types of systems are often categorized as either continuum or statistical and a broad range of approaches exist (Sahimi et al., 1990). While many methods to model these reactive transport processes in porous media have been developed, data on the alteration of pore scale dynamics due to pore structure altering reactions are limited.

Nuclear magnetic resonance (NMR) methods have proven to provide unique data on displacement length and timescale dynamics of hydrodynamic dispersion for flow in three dimensional (3D) porous media (Brosten et al., 2009; Lebon et al., 1996; Manz et al., 1999b; Scheven et al., 2007; Seymour and Callaghan, 1997). Another class of pore structure evolving reactions in porous media (Clement et al., 1996; Thullner and Baveye, 2008) to which NMR has been applied is biofouling, i.e. biomass generating reactive flows. NMR data 
have characterized the interaction between pore structure evolution due to biomass growth and the pore dynamics of hydrodynamic dispersion (Graf von der Schulenberg et al., 2008; Seymour et al., 2004, 2007). In particular NMR has provided data indicating the transition from normal diffusive transport to anomalous diffusive transport due to the alteration of the pore structure by biomass growth from homogeneous to heterogeneous (Seymour et al., 2004, 2007). In this paper NMR measurements of the hydrodynamic dispersion in a model porous structure before and after a biofilm mediated precipitation reaction are presented. It is shown that the precipitation reaction deposits a layer of solid precipitate that induces a more rapid transition toward asymptotic Gaussian hydrodynamic dispersion dynamics, in contrast to the anomalous non-Gaussian dynamics observed for biofouling by a microbe that produces significant extracellular polymeric substance but not a solid precipitate.

\section{Theory}

\subsection{Hydrodynamic dispersion}

Due to the extensive nature of the literature on reactive transport and hydrodynamic dispersion models for porous media, a limited discussion of the applicable theory is provided only to lend context to the discussion of the NMR data. Models are often delineated into categories as statistical or continuum based (Sahimi et al., 1990). This demarcation is somewhat artificial due to the fact that the advection diffusion equation (ADE) can be derived from concepts of both statistical mechanics (Mazo, 1967) and continuum mechanics (Bear, 1972; Bird et al., 1960). The ADE

$\frac{\partial P(Z, t)}{\partial t}=\left[-\left\langle v_{z}\right\rangle \frac{\partial}{\partial Z}+D^{*} \frac{\partial^{2}}{\partial Z^{2}}\right] P(Z, t)+R$

gives the time rate of change of probability $P(Z, t)$, which is equivalent to normalized concentration, due to advection by a mean flow $\left\langle v_{\mathrm{z}}>\right.$ and diffusive spreading due to hydrodynamic dispersion $D^{*}$. The ADE in Eq. (1) is the one dimensional averaged mass conservation equation valid over a spatial scale of many pores, or even the entire system, where the pore scale is no longer resolved. In Eq. (1) $\left\langle v_{z}\right\rangle$ is the appropriately averaged velocity and $D^{*}$ the asymptotic hydrodynamic dispersion coefficient. The appropriate form of the reactive source/sink term $R$ in Eq. (1) for reactive flows is an area of intense research interest (Emmanuel and Berkowitz, 2005, 2007; Tartakovsky et al., 2007). However, as discussed below, the NMR data we acquire compares the hydrodynamic dispersion dynamics before and after a pore structure altering reaction event, so our primary interest is in the dynamics not the reaction rate behavior of $R$.

The ADE generates a normal diffusion process in which the dynamics are Gaussian in the long time limit relative to mixing over many pore lengths. Hydrodynamic dispersion processes occurring at a short time scale, i.e. short displacement length-scale relative to pore size, are non-Gaussian due to correlated dynamics on the pore scale. In other words, the preasymptotic dynamics reflect the process of the decay of correlation of dynamics due to fluctuations in velocity by mechanical streamline mixing, diffusion across streamlines and hold up in dead end pores (Salles et al., 1993). The pore structure of the porous media determines the impact of the different dispersion mechanisms and controls the approach of the dynamics to the asymptotic Gaussian behavior. Nonequilibrium statistical mechanics models based on memory function equations (Boon and Yip, 1991) for the time and length-scale dependence of preasymptotic dynamics have been developed (Cushman et al., 1994) and elucidated by PGSE NMR measurements (Brosten et al., 2009). In heterogeneous pore structure media, where nonGaussian anomalous diffusion scale dependent transport is present for asymptotic times, continuous time random walk (CTRW) models (Berkowitz et al., 2006) and non-local continuum approaches (Koch and Brady, 1988) result in fractional advection diffusion equations (FADE) (Cushman and Ginn, 2000). The applicability of such models to reactive systems with pore evolution has not been demonstrated.

\subsection{NMR measurement of dispersion}

NMR provides unique data on transport in porous media due to the ability to noninvasively resolve dynamics within the pore structure. The measurement of dynamics as a function of varying displacement length and time generates data on a hierarchy of displacement scales. The acquired NMR signal using a standard pulsed gradient spin echo (PGSE) method shown in Fig. 1 is encoded in the experimental Fourier domain reciprocal to displacement in terms of the wave vector $q=(2 \pi)^{-1} \gamma g \delta$ and the displacement observation time $\Delta$ (Blümich, 2000; Callaghan, 1991). In this way the dynamics are measured as a function of displacement observation time $\Delta$ and varying displacement length-scale $q^{-1}$. The measured voltage signal in the NMR experiment is given by

$E(q, \Delta)=S(q, \Delta) / S(q=0, \Delta)=\int P(Z, \Delta) \exp [i 2 \pi q Z] d Z$.

where $Z=Z^{\prime}(\Delta)-z(0)$ is the displacement of the NMR active nuclei, or spins, and $P(Z, \Delta)=\int \rho(x, y, z) P_{s}\left(z, 0 \mid z^{\prime}, \Delta\right) d x d y d z$ is the averaged propagator. There is a Fourier transform relationship between the echo signal $E(q, \Delta)$ acquired by incrementing the gradient amplitude $g$ applied for time $\delta$ and the average propagator. The averaged propagator, or van Hove self-correlation function (Boon and Yip, 1991), is the conditional probability $P_{\mathrm{s}}\left(z, 0 \mid z^{\prime}, \Delta\right)$ that a spin residing at $z$ at time 0 moves to $z^{\prime}$ at time $\Delta$ averaged over the initial spin distribution $\rho(x, y, z)$ (Callaghan, 1991). Complete details of the dynamics are contained within the propagator for all displacement scales sampled by $q$.

NMR imaging (MRI) has limited spatial resolution since NMR is an inherently low signal to noise technique based on the small differences in energy state populations of the spins. Even under ideal conditions in a porous column of size of the order of $10 \mathrm{~mm}$ the spatial resolution of MRI is limited to about $(20 \mu \mathrm{m})^{3}$, rendering MRI methods of limited effectiveness at measuring pore scale dynamics. Additionally, at this scale, most MRI velocity methods require many minutes to obtain a spatially resolved map of velocity. Specially designed rapid MRI velocity methods are capable of acquiring spatial distributions of velocity in as little as $50 \mathrm{~ms}$ and have potential to provide data on variations in flow as reactions 


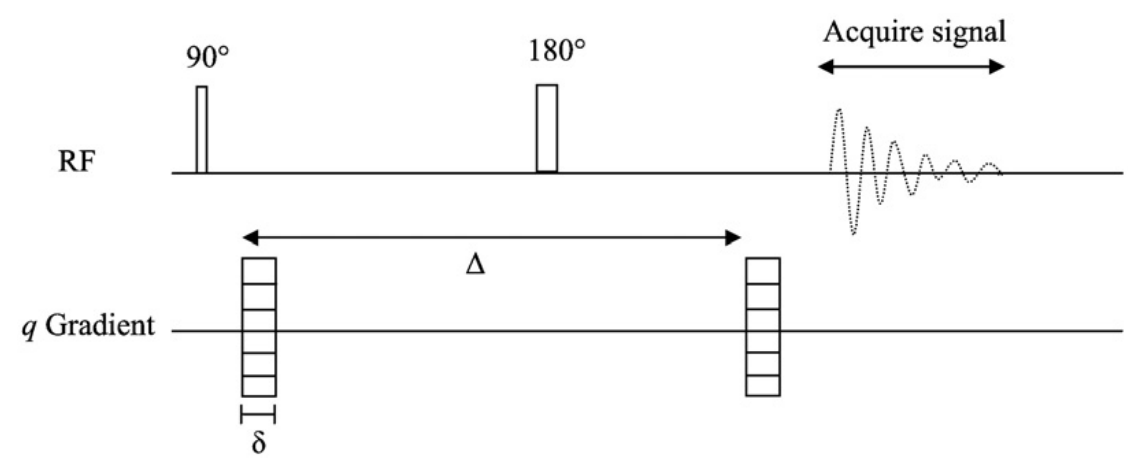

Fig. 1. Pulsed gradient spin echo (PGSE) NMR pulse sequence used to generate propagators of motion over a range of displacement lengths reciprocal to the experimentally incremented wave vector $q=(2 \pi)^{-1} \gamma g \delta$ and displacement observation time $\Delta$.

are occurring (Kose, 1994; Sederman et al., 2004), but these methods are typically limited to spatial resolutions greater than $(100 \mu \mathrm{m})^{3}$ due to signal to noise considerations. The strength of the MRI method, as presented here, is the ability to probe the impact of coarse graining space on measured translational and rotational molecular dynamics. As stated above, the pore scale dynamics induced by the structure of the porous media is contained within the scale dependent dynamics measured by the non-spatially resolved propagator without the need for the use of imaging methods (Callaghan, 1991; Callaghan et al., 1991; Seymour and Callaghan, 1997). Hence, the propagators presented here allow for the characterization of the pore scale dynamics and hence a deduction of changes that are occurring at the pore scale even without pore scale spatial resolution of the data.

\section{Experimental details}

\subsection{Model bead pack and microbiology}

A $10 \mathrm{~mm}$ ID and $30 \mathrm{~mm}$ length liquid chromatography column (Omnifit) with a frit at the entrance and $220 \mu \mathrm{m}$ mesh at the exit was packed with $d_{\mathrm{p}}=241 \mu \mathrm{m}$ diameter monodisperse polystyrene beads (Duke Scientific 4324A). After assembly the porosity was measured to be $\phi=0.41$ by analyzing NMR images of the bead pack and confirmed by the measurements of the mean velocity inside the bead pack and total volumetric flow rate through the bead pack. Experiments utilized a dual syringe HPLC pump (Pharmacia P-500) which allows controlled volumetric flow rates. DI water, media without and with calcium was pumped through the bead pack against gravity to a reservoir above the column. The DI water was doped with Magnevist to allow for a shortened NMR experimental repetition time $T_{R}$ this was unnecessary for the medium with and without calcium as the salts and sugars reduce the spin-lattice $\mathrm{T}_{1}$ magnetic relaxation time. NMR measurements were conducted for the flow of these three fluids through the clean bead pack (CBP) to acquire baseline results for later comparison with the inoculated and precipitated bead pack (IBP).

For the experiments, the column was inoculated with a culture of the ureolytically active bacterium Sporosarcina pasteurii (formerly classified as Bacillus pasteurii) in the growth medium described by Ferris and Stehmeier (1996).
The medium includes $3 \mathrm{~g} / \mathrm{L}$ nutrient broth (Becton, Dickinson and Company), $20 \mathrm{~g} / \mathrm{L}$ urea (Fisher Scientific), $10 \mathrm{~g} / \mathrm{L}$ ammonium chloride (Fisher Scientific), and $2.1 \mathrm{~g} / \mathrm{L}$ sodium bicarbonate (Fisher Scientific). Calcium chloride dihydrate (Acros Organics) was added to the medium at a concentration of $3.7 \mathrm{~g} / \mathrm{L}$ when media was desired with sufficiently high calcium concentration for precipitation to occur. In the process of bacterially facilitated urea hydrolysis (ureolysis), the production of ammonium and dissolved inorganic carbon during decomposition of urea by the bacteria increases the solution $\mathrm{pH}$ and carbonate concentration, which increases saturation and favors calcium carbonate precipitation. The reaction mechanism is illustrated in the following equations (Ferris et al., 2003):

$\mathrm{CO}\left(\mathrm{NH}_{2}\right)_{2}+\mathrm{H}_{2} \mathrm{O} \rightarrow \mathrm{NH}_{2} \mathrm{COOH}+\mathrm{NH}_{3} \rightarrow 2 \mathrm{NH}_{3}+\mathrm{CO}_{2}$ (urea hydrolysis)

$2 \mathrm{NH}_{3}+2 \mathrm{H}_{2} \mathrm{O} \leftarrow \rightarrow 2 \mathrm{NH}_{4}^{+}+2 \mathrm{OH}^{-}$(pH increase)

$\mathrm{CO}_{2}+2 \mathrm{OH}^{-} \leftarrow \rightarrow \mathrm{CO}_{3}^{2-}+\mathrm{H}_{2} \mathrm{O}$ (carbonate formation)

$\mathrm{CO}_{3}^{2-}+\mathrm{Ca}^{2+} \leftarrow \rightarrow \mathrm{CaCO}_{3 \text { (solid) }}$ (precipitation)

While the hydrodynamic alterations resulting from calcium carbonate precipitation in the porous medium were of primary interest in this experiment work is ongoing to further correlate the reaction kinetics to the changes in hydrodynamics.

\subsection{NMR experiments}

The bead pack was secured in a $20 \mathrm{~mm}$ ID radio frequency (rf) coil and placed inside a $300 \mathrm{MHz}$ vertical standard bore superconducting magnet. A Bruker Avance III spectrometer was networked to the superconducting magnet. A Bruker Micro2.5 microimaging probe and gradient amplifiers were used which allowed imaging using gradients up to $1.48 \mathrm{~T} / \mathrm{m}$ in all three directions. PGSE experiments were conducted to acquire propagator data on the probability of spin displacement and q-space data to measure any diffraction effects. Additionally, standard imaging sequences were used to 
acquire images through the whole bead pack and $\mathrm{T}_{2}$ maps of slices through the bead pack. The experimental parameters for these different experiments are listed below:

PGSE experiments: acq-pts $=512$, q-pts $=130$, slice thickness: $1 \mathrm{~mm}, \mathrm{~T}_{\mathrm{R}}=2 \mathrm{~s}, \delta=2 \mathrm{~ms}, \Delta=[25,50, \ldots, 600] \mathrm{ms}$, $\mathrm{g}_{\max }=0.19-1.04 \mathrm{~T} / \mathrm{m}, \mathrm{SW}=50 \mathrm{kHz}, \mathrm{N}_{\mathrm{A}}=8$. Experimental time $=35 \mathrm{~min}$.

T2 imaging experiments: pixels $=256 \times 256, \mathrm{FOV}=11 \times 11 \mathrm{~mm}^{2}$, Voxel resolution: $43 \times 43 \times 2000 \mu \mathrm{m}^{3}, \mathrm{~T}_{\mathrm{E}}=[10,20, \ldots 160] \mathrm{ms}$, $\mathrm{T}_{\mathrm{R}}=1 \mathrm{~s}, \mathrm{SW}=50 \mathrm{kHz}, \mathrm{N}_{\mathrm{A}}=8$, Experimental time $=34 \mathrm{~min}$.

NMR imaging experiments ( 5 slices): pixels $=256 \times 256$, FOV $=30 \times 11 \mathrm{~mm}^{2}$, voxel resolution: $117 \times 43 \times 2000 \mu^{3}$, $\mathrm{T}_{\mathrm{E}}=14 \mathrm{~ms}, \mathrm{~T}_{\mathrm{R}}=1 \mathrm{~s}, \mathrm{SW}=50 \mathrm{kHz}, \mathrm{N}_{\mathrm{A}}=1$, Experimental time $=4 \mathrm{~min}$.
After NMR measurements had been conducted on the clean bead pack (CBP), the column was inoculated by adding $6.5 \mathrm{~mL}$ of a $S$. pasteurii culture to the top of the column and reversing flow through the column at $10 \mathrm{~mL} / \mathrm{h}$. The bacteria were allowed to attach for $60 \mathrm{~min}$, before the flow of calciumfree medium was begun. Medium flow was continued for $60 \mathrm{~min}$ before MRI measurements were initiated, which required no-flow conditions. Three MRI measurements were performed approximately $4 \mathrm{~h}$ after inoculation, before calcium-containing medium was pumped through the bead pack at $400 \mathrm{~mL} / \mathrm{h}$ for $10 \mathrm{~min}$. PGSE NMR and MRI measurements were performed. Additional injection of media with calcium was done after about $5 \mathrm{~h}$ of NMR experiments. Between NMR experiments calcium-free medium was pumped through the column at $10 \mathrm{~mL} / \mathrm{h}$ to maintain nutrient
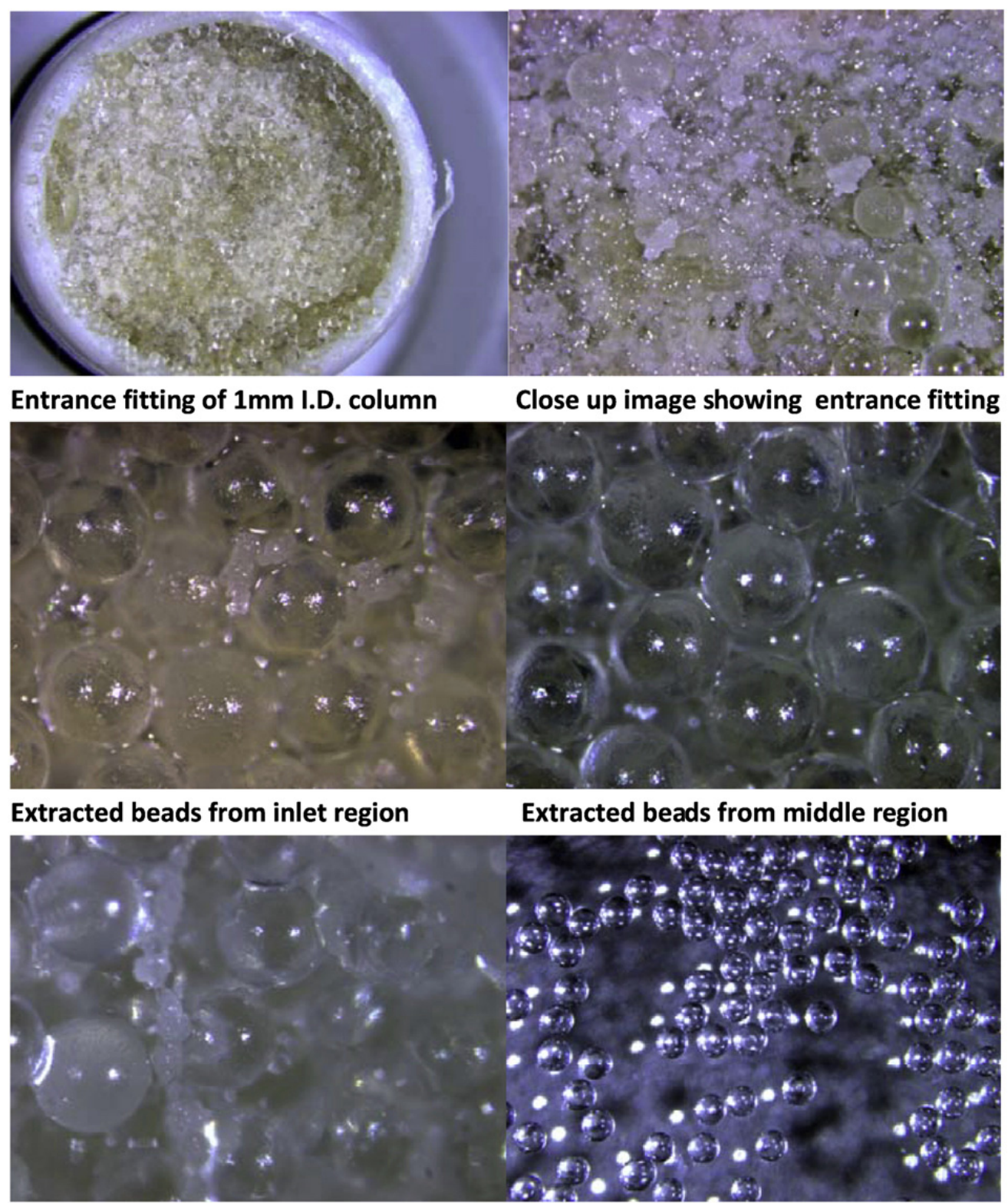

Extracted beads from outlet region

Image of clean spheres prior to use.

Fig. 2. Selected stereo microscope (Nikon SMZ1500) images of beads from different regions of the porous media column showing the deposition of biomass and precipitate. The diameter of each bead is $241 \mu \mathrm{m}$, providing a scale for each image. 


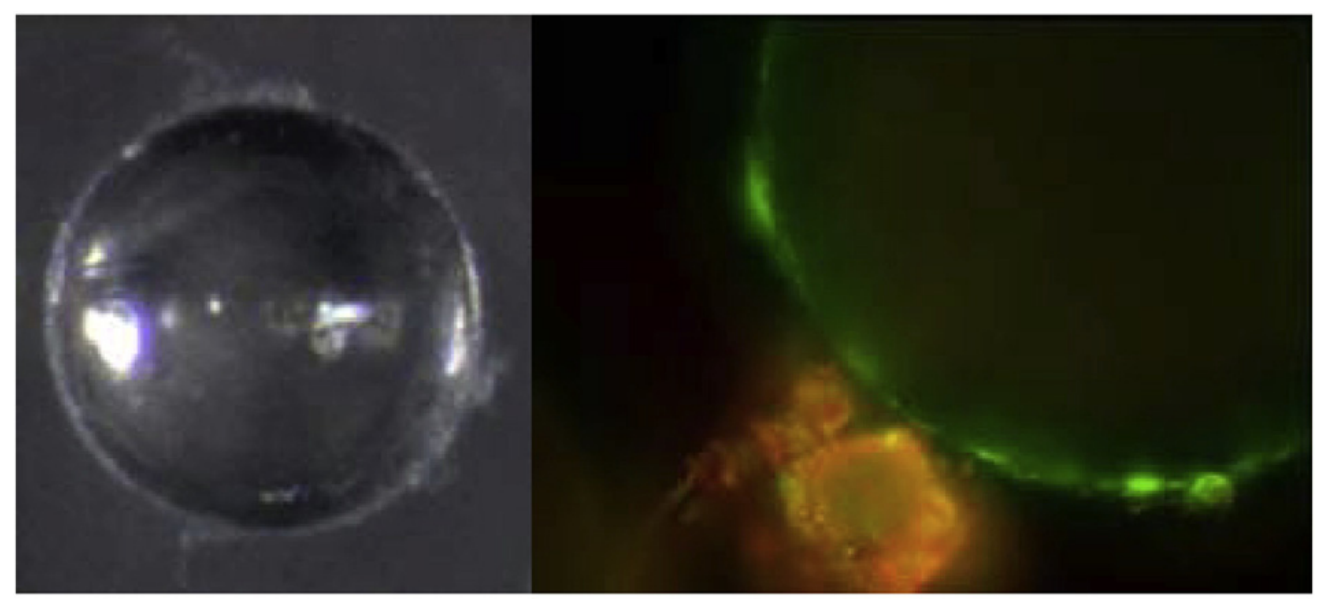

Fig. 3. A stereo microscope image (left) of biomass attached to a $241 \mu \mathrm{m}$ diameter sphere that was extracted from the column. This bead was gently treated with $10 \%$ nitric acid $\left(\mathrm{HNO}_{3}\right)$ to dissolve the precipitates and visualize only attached biomass. A fluorescent microscope image (Nikon Eclipse E800, 40× objective) (right) of an extracted sample that was stained with Syto 9 and Propidium iodide (Invitrogen LIVE/DEADß). Syto 9 (green) highlights the bacteria with intact cellular membranes, which appear to be at a higher density surrounding the bead. Propidium iodide (red) highlights cells with compromised membranes. A precipitate crystal is highlighted by the propidium iodide. This is due to either a higher density of cells with compromised membranes surrounding the precipitate and/or staining of the organic matrix associated with the calcium carbonate by the propidium iodide.

supply for bacterial growth. All PGSE NMR experiments were conducted at a flow rate of $400 \mathrm{~mL} / \mathrm{h}$. The ureolytic activity of the bacteria was assessed by acquiring fluid samples at the exit of the magnet (approximately $1 \mathrm{~m}$ from the column exit) and measuring the $\mathrm{pH}$ value as a function of flow rate.
In calcium-free systems, S. pasteurii was unable to form thick biofilms and affect the hydrodynamics in porous media even after several days (data not shown). The changes in hydrodynamics induced over the roughly 24 hour period described here, are caused by a combination of mineral

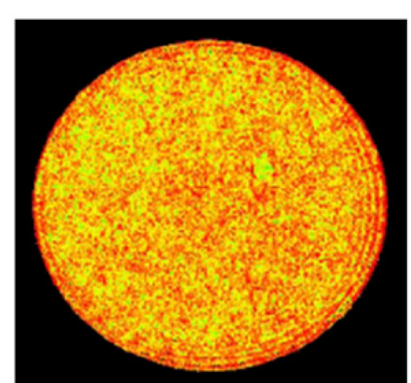

M: 54.86

SD: 2.78

$\underline{T}=\mathbf{0 m i n}$

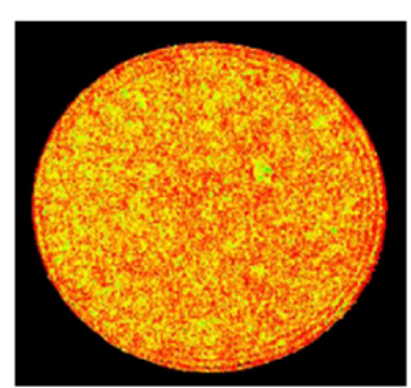

M: 55.42

SD: 2.75

$\mathrm{T}=260 \mathrm{~min}$

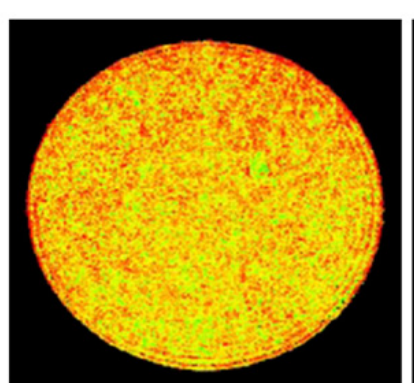

M: 53.94

SD: 2.69

$\mathrm{T}=66 \mathrm{~min}$

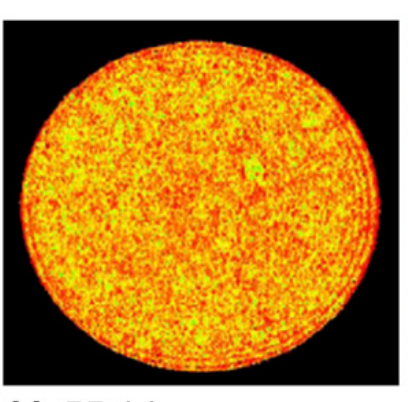

M: 55.14

SD: 2.75

$T=130 \mathrm{~min}$

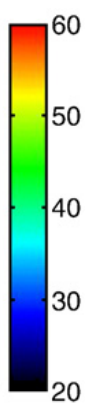
西 
precipitation and biomass accumulation. The fastest doubling time observed for this strain in our laboratories during $\mathrm{CaCO}_{3}$ precipitation in continuously shaken batch systems was $5 \mathrm{~h}$ or longer. Microbial cells in biofilms are usually diffusion limited and hence growth rates in the column are likely to be lower than observed in batch experiments. Hence, significant biomass accumulation likely did not occur and $\mathrm{CaCO}_{3}$ mineral precipitation was most likely the major contributor towards changes in pore structure and hydrodynamics in the column.

\section{Results}

\subsection{Optical images and MRI}

Stereo microscope (Nikon SMZ1500) optical images of the beads extracted from various locations in the packed column after the experimental run are shown in Fig. 2. The images indicate an axial variation in the amount of precipitate and biomass. The entrance fitting shows significant deposition. The bottom of the column, the flow inlet region, and the top flow outlet of the column appear similar while the middle region where the PGSE NMR measurements are localized has slightly less precipitate and biomass. Fig. 3a shows a stereo microscope image of a single sphere in which a deposition layer is apparent on the sphere surface. A fluorescent microscope image (Fig. 3b) indicates that the deposition material in the images of Fig. 2 is composed of attached biomass, stained green and $\mathrm{CaCO}_{3}$ precipitate crystals, visible in orange/red. The microscope images in Figs. 2 and 3 indicate the deposition of precipitate and biomass in pores between spheres as well as fairly uniform coating around the spheres. Interestingly $\mathrm{T}_{2}$ spin-spin magnetic relaxation maps (Blümich, 2000; Callaghan, 1991) in Fig. 4 show little or no impact from the precipitate and biomass. This is in contrast to NMR studies of thick biofilm forming bacteria which give significant $T_{2}$ relaxation variations in porous media due to the impact of the extracellular polymeric substance (EPS) on the rotational mobility of water molecules constrained within the EPS hydrogel (Hoskins et al., 1999; Seymour et al., 2004). This is attributable to the fact that most of the pore blockage evident in Fig. 2 is $\mathrm{CaCO}_{3}$ with thin layers of biomass as shown in Fig. 3. The $\mathrm{CaCO}_{3}$ reduces the amount of pore water but in contrast to the presence of biofilm (Seymour et al., 2004, 2007) does not significantly impact the $T_{2}$ relaxation time of the remaining pore water. MRI images of the signal intensity in the packed column as a function of the reaction process are shown in Fig. 5. As mentioned above the spatial resolution averages signal intensity over multiple pores. In the data shown, the volume elements over which the averaging occurs, i.e. the spatial resolution of the images, are $117 \mu \mathrm{m}$ by $43 \mu \mathrm{m}$ in plane over $2 \mathrm{~mm}$ slice thickness. Two features are apparent: First a region of increased signal intensity near the flow entrance on one side

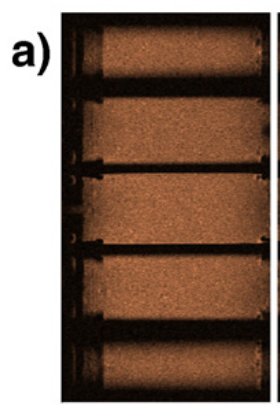

$\mathrm{T}_{1}=37 \mathrm{~min}$

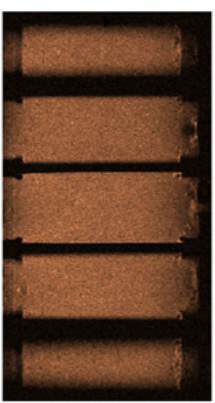

$\mathrm{T}_{1}=42 \mathrm{~min}$

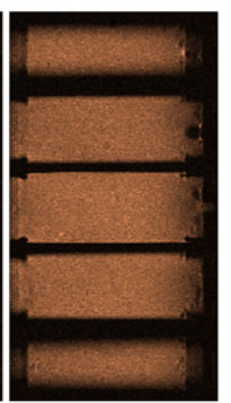

$\mathrm{T}_{1}=57 \mathrm{~min}$

Before addition of Calcium

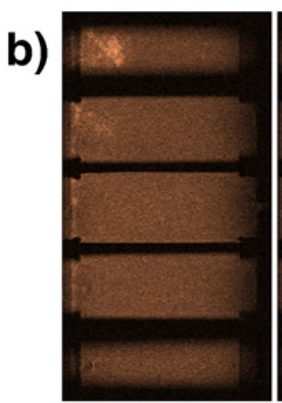

$T_{1}=262 \mathrm{~min}$ After addition

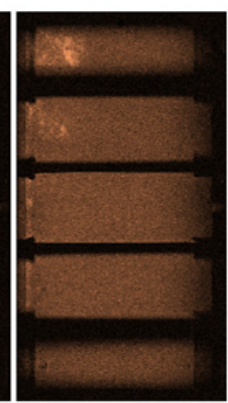

$\mathrm{T}_{1}=276 \mathrm{~min}$

of Calcium

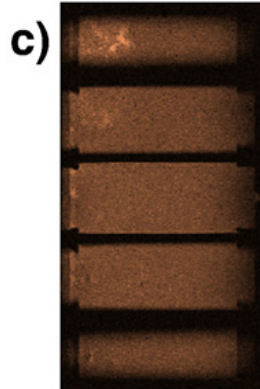

$\mathrm{T}_{1}=307 \mathrm{~min}$ $\mathbf{T}=0 \mathrm{~min}$

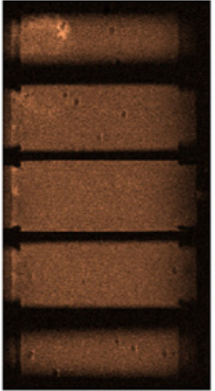

$T_{1}=338 \mathrm{~min}$ $T=31 \mathrm{~min}$

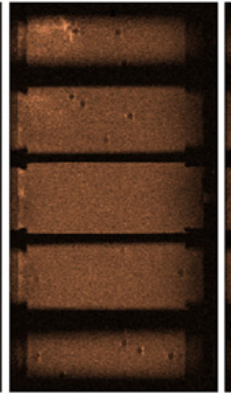

$\mathrm{T}_{1}=350 \mathrm{~min}$ $T=43 \mathrm{~min}$

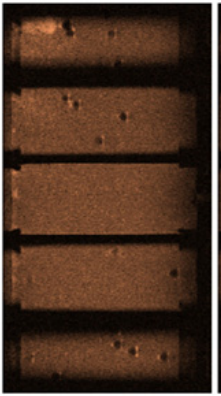

$\mathrm{T}_{1}=390 \mathrm{~min}$ $\mathbf{T}=83 \mathrm{~min}$

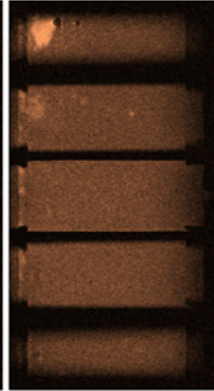

$T_{1}=432 \mathrm{~min}$ $\mathbf{T}=\mathbf{0} \mathrm{min}$

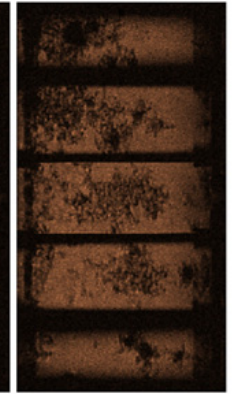

$T_{1}=1438 \mathrm{~min}$ $\mathrm{T}=1006 \mathrm{~min}$

Fig. 5. A series of MRI's images of five $2 \mathrm{~mm}$ thickness slices across column. $\mathrm{T}_{\mathrm{I}}$ is the time after inoculation and $\mathrm{T}$ is the elapsed time for a particular experimental series. a) Images obtained after inoculation, before any flow experiments. b) Images obtained after flow of calcium-containing medium for $10 \mathrm{~min}$ at $400 \mathrm{~mL} / \mathrm{h}$. These show lower signal intensity due to presence of calcium in column and a bright region close to the wall (top two images). c) Images obtained after flow of calcium-containing medium for $24 \mathrm{~min}$ at $400 \mathrm{~mL} / \mathrm{h}$, showing growth of dark spots over time. After $\mathrm{T}_{\mathrm{I}}=390 \mathrm{~min}$ the column was flushed with calcium-free medium at $400 \mathrm{~mL} / \mathrm{h}$ resulting in removal of dark spots $\left(\mathrm{T}_{\mathrm{I}}=432 \mathrm{~min}\right)$. Then the column was left to rest for $\sim 20 \mathrm{~h}$, resulting in the re-growth of dark spots over time $\left(\mathrm{T}_{\mathrm{I}}=1438 \mathrm{~min}\right)$. Between MR Images calcium-free medium was pumped through the column at $10 \mathrm{~mL} / \mathrm{h}$ to maintain nutrient supply for bacterial growth. The column was flushed again with calcium-free medium at $400 \mathrm{~mL} / \mathrm{h}$ before further experiments were conducted. MRI parameters ( 5 slices): $256 \times 256$ points, Voxel resolution: $117 \times 43 \times 2000 \mu \mathrm{m}, \mathrm{FOV}=30 \times 11 \mathrm{~mm}, \mathrm{~T}_{\mathrm{E}}=14 \mathrm{~ms}, \mathrm{~T}_{\mathrm{R}}=1 \mathrm{~s}, \mathrm{SW}: 50 \mathrm{kHz}$. 
of the column after the introduction of $\mathrm{Ca}$ which persists throughout the experiment; second the appearance of low signal intensity potentially due to the generation of gas through bacterial growth. No significant details regarding the reaction process on the pore structure is evident, again showing the limitation of direct NMR imaging to provide significant information on pore evolution.

\subsection{PGSE NMR measurements}

In contrast to the limited information provided by spatially resolved NMR the measurement of dynamics is highly sensitive to the reaction induced pore structure change (Seymour et al., 2004, 2007). Fig. 6 shows the displacement time $\Delta$ dependence of the dynamics as given by the propagator for a $400 \mathrm{~mL} / \mathrm{h}$ flow rate before (Fig. 6a) and after (Fig. 6b) reaction. The clean bead pack (Fig. 6a) indicates the well known progression of the dynamics from the preasymptotic regime with a Poisson like distribution of displacements within single pores at short displacement time $\Delta=25 \mathrm{~ms}$ to near Gaussian behavior at the longest observation time $\Delta=600 \mathrm{~ms}$. Interestingly the post reaction dynamics (Fig. 6b) show a similar general progression with
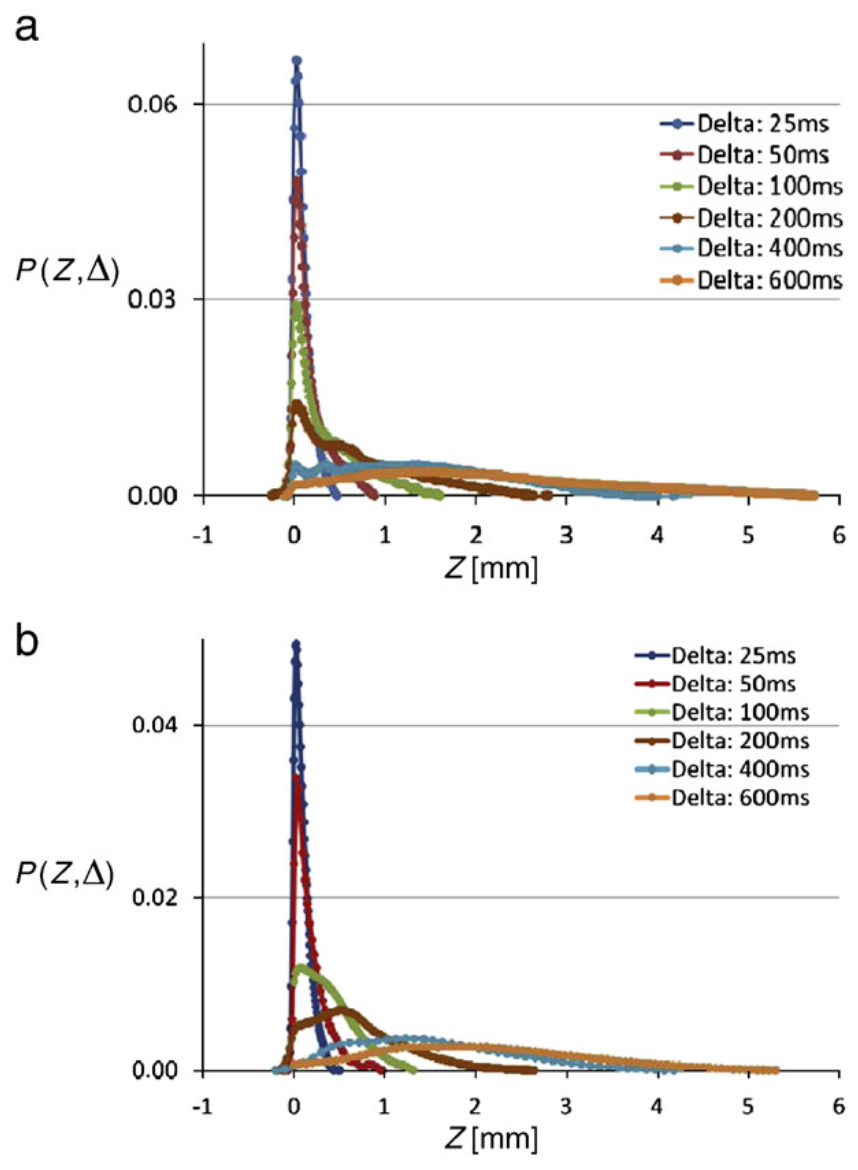

Fig. 6. Propagators as a function of displacement observation time for $400 \mathrm{~mL} / \mathrm{h}$ flow of media without calcium in a) the clean bead pack and b) the inoculated bead pack after precipitation. displacement time. However, closer inspection of the propagators at each time $\Delta$, shown in Fig. 7, indicates significant differences in the dynamics on the pore scale. At displacement observation time $\Delta=25 \mathrm{~ms}$ the water molecules are within a pore and the propagator reflects the distribution of velocity within the pores. Over this time little or no Taylor dispersion due to diffusion across streamlines occurs since the diffusion length $l_{\mathrm{D}}=\left(2 D_{\mathrm{o}} \Delta\right)^{1 / 2} \sim 10 \mu \mathrm{m}$ is a small fraction of the pore size $l_{\mathrm{p}}=d_{\mathrm{p}} \phi /(1-\phi) \sim 167 \mu \mathrm{m}$ based on the clean bead pack (CBP). Mechanical mixing due to streamline crossing induced by the tortuous flowpath was not observed either, since the time to transit a pore based on the superficial velocity $\langle v\rangle=3.45 \mathrm{~mm} / \mathrm{s}$ is $\tau_{v}=l_{\mathrm{p}} /\langle v>\sim 48 \mathrm{~ms}$. Despite the precipitate and biomass evident in Figs. 2 and 3 the pore scale distribution of displacements due to velocity within a pore are nearly the same. At displacement time $\Delta=50 \mathrm{~ms}$ the molecules have just transited a single pore on average and the distribution of displacements is still quite similar, however some variation at displacements on the order of one or two pore lengths $l_{\mathrm{p}}$ is beginning to be evident.

Once the displacement observation time is such that significant dispersion due to sampling of multiple pores begins to occur, $\Delta=100 \mathrm{~ms}$, a significant change in the dynamics between the CBP and the inoculated and precipitated bead pack (IBP) occur. The CBP still exhibits a strong peak at small displacements on the order of the pore length while the displacement distribution for the IBP has a sizeable number of molecules with intermediate displacements. Of interest are the absence of a large displacement tail in the IBP indicating that significant channeling is not occurring and the onset of oscillations on the CBP displacement probability due to correlations in motion from the ordering of the monodisperse packing of the CBP generating a diffraction like effect in the $q$-space data (Manz et al., 1999a; Seymour and Callaghan, 1997; Seymour et al., 2004, 2007). Analogous differences are present at $\Delta=200 \mathrm{~ms}$. By displacement time $\Delta=400 \mathrm{~ms}$ the oscillations due to the homogeneous structure of the CBP are clearly evident and the CBP maintains a larger distribution of molecules at small displacements. The dispersion in the IBP is actually smaller than the CBP as evidenced by the narrower distribution of displacements. The distributions at displacements greater than about $1.7 \mathrm{~mm}$ are the same indicating a similarity in the dispersion dynamics after molecular sampling of 10 pore lengths. These observations re-emphasize the strength of PGSE NMR measurements since potentially important changes in pore structure can be directly revealed, which cannot be detected by traditional (dye) tracer measurements, MRI, or other methods, which require averaging over larger time or length scales. The data directly quantify the changes in dynamics that define the time and length scales of molecular displacements associated with the transition from preasymptotic to asymptotic behavior. The correlated motions of molecules responsible for the oscillations and higher probability of smaller displacements on the CBP propagators indicate the longer duration of the preasymptotic regime, defined in terms of the presence of correlated dynamics, in the CBP relative to the IBP. At the longest displacement observation time $\Delta=600 \mathrm{~ms}$ the IBP dynamics are more narrowly distributed around the mean displacement and again more molecules have small displacements in the CBP. This persistence of a larger distribution of 

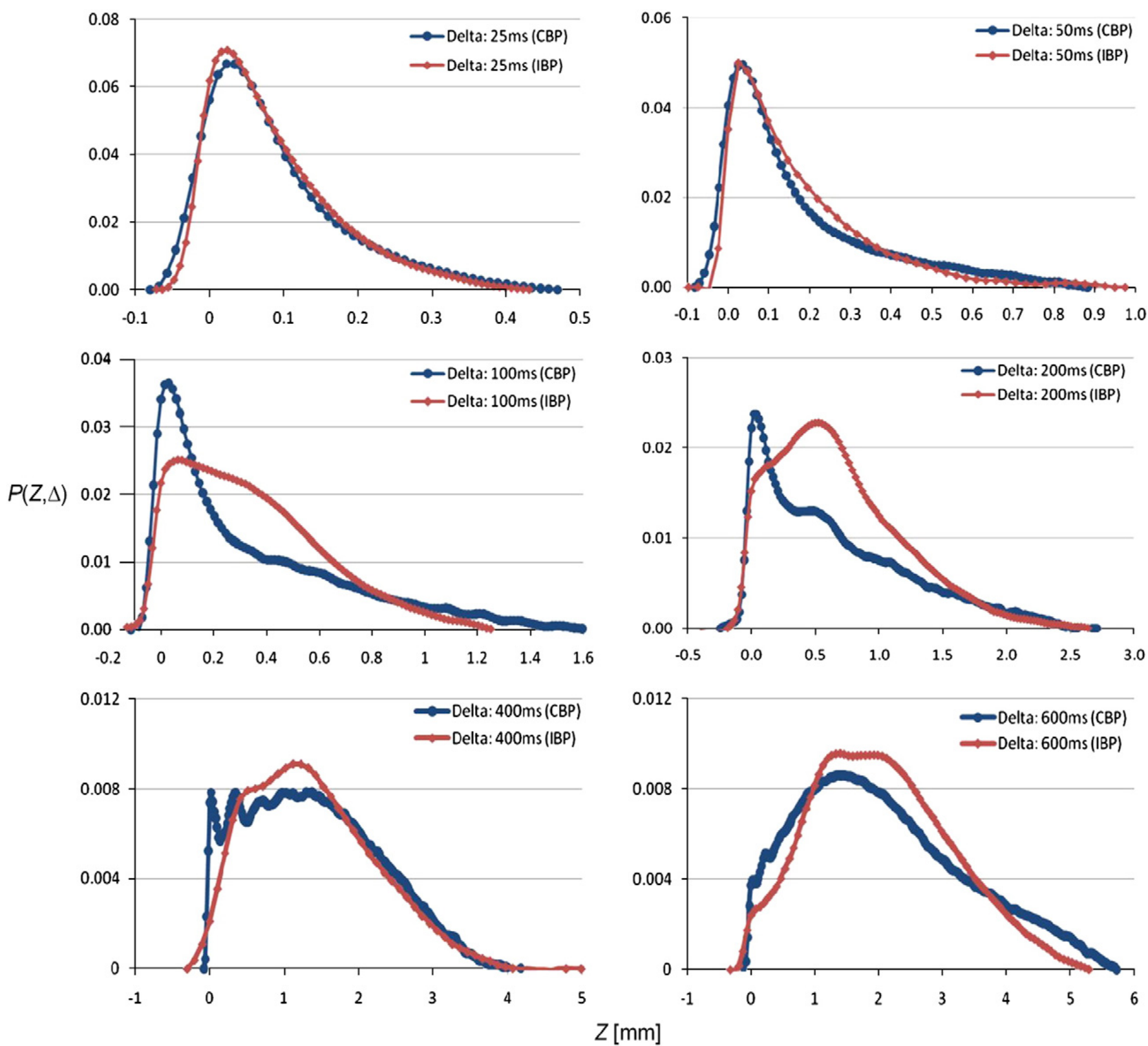

Fig. 7. Comparison of clean bead back (CBP/blue or black) and inoculated bead pack (IBP/red or grey) for displacement observations times $\Delta$ shown on each figure.

molecules with small displacements indicates that rather than the precipitation reaction generating significant dead end pores or greater surface area for no slip condition stagnant molecules, the precipitate is decreasing the hold up of molecules. Coupled to the narrower distribution of dynamics in the IBP this indicates that the precipitate alters the pore structure such that microscale mixing is enhanced allowing the displacements to be more homogeneous. The precipitate is generating a rough and irregular surface at the micro scale smaller than a pore length, or bead diameter, which alters the dynamics at intermediate displacement scales by mechanical mixing of streamlines near pore walls in contrast to the smooth and ordered CBP. At the longest observation time the distribution of displacements for the reacted IBP is approaching a Gaussian distribution more rapidly than the $\mathrm{CBP}$, including fewer molecules undergoing displacements greater than $4 \mathrm{~mm}$.
Despite the fact that all the information on the dynamics is present in the propagator, in considering the impact of the precipitation altered pore structure on the dynamics it is useful to look at the propagator data in the reciprocal qspace. Fig. 8 shows data for $\Delta=400 \mathrm{~ms}$ for the CBP with deionized (DI) water and media flowing through it, as well as data for flow of media without $\mathrm{Ca}$ as the reaction proceeded in the IBP after inoculation and injection of Ca enriched media. Data shown for the IBP is taken every $5 \mathrm{~min}$ as the reaction proceeds. Note the increase in the rate of signal decay for the IBP relative to the CBP at low $q$, corresponding to large displacements. This indicates the more rapid approach to Gaussian behavior and more rapid loss of correlation in displacement dynamics in the reactive transport IBP system. At this observation time the coherent diffraction peak at the characteristic pore structure size (Callaghan et al., 1999) is evident for both the CBP and the 


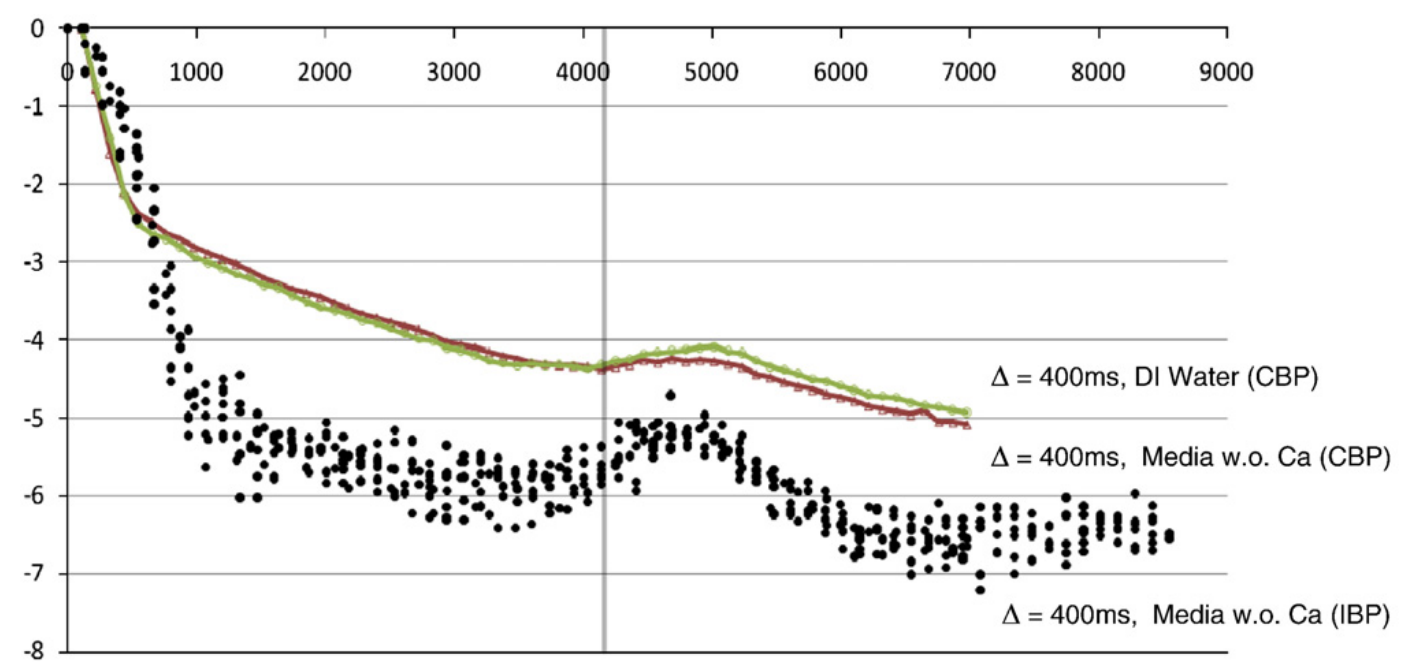

Fig. 8. PGSE data plotted in $q$-space showing the diffraction effect due to the ordered structure of the porous media for the clean bead back (CBP) and inoculated and precipitated bead pack (IBP) at displacement observation time of $\Delta=400 \mathrm{~ms}$. The data for the IBP taken over several hours after precipitation has occurred indicates that while there is increased noise due to the pore structure evolving the dynamics are consistent over a long time period.

IBP. In the IBP the maxima and minima of the diffraction peak are broadened and shifted to slightly lower values of $q$ indicating a broader distribution of pore sizes in the IBP due to the reactive change in the pore structure. The IBP data is noisy but consistent over many hours of observation of the flow. The precipitate is not fully blocking the pores and increasing hold up dispersion in dead end pores which would generate large displacements due to channeling. Rather the precipitate alters the pore structure more subtly by coarsening the surfaces and broadening the pore size distribution so that mixing and dispersion within pores is enhanced but longer range structure is not lost as evidenced by the coherent diffraction effects in the $q$-space data.

\section{Conclusions}

NMR measurements of a homogeneous model porous medium of $241 \mu \mathrm{m}$ polymer beads were carried out before and after a biologically mediated precipitation reaction in order to explore the impact of reactive pore structure alteration on transport dynamics. MRI data is shown to be of limited use in analyzing these systems based on limitations in spatial resolution. In contrast, PGSE NMR measurements allowed for the acquisition of propagator data as a function of displacement time (and length), which clearly indicated alteration of the pore structure. The precipitation reaction alters the pore structure relative to a clean bead pack such that fewer molecules undergo small displacements within individual pores over a range of displacement times and generates a narrower distribution of dynamics which approaches a Gaussian distribution more rapidly. The pore surfaces of the reactive IBP were roughened and enhanced mixing and mechanical dispersion on the pore scale resulting in the observed dynamics. Integration of such information into conceptual and mathematical models has the potential to improve quantitative predictions of the impact of reactive processes on flow and transport in porous media.

\section{Acknowledgements}

This research was supported in part by the Office of Science (BER) U.S. Department of Energy DE-FG0207ER64416 (JDS and SLC), the US Department of Energy EPSCoR program grant number DE-FG02-08ER46527 (ABC) and the National Science Foundation DMS-0934696 (RG). Equipment funding was provided by the NSF MRI and the M.J. Murdock Charitable Trust (JDS and SLC).

\section{References}

Bear, J., 1972. Dynamics of Fluids in Porous Media. American Elsevier, New York.

Berkowitz, B., Cortis, A., Dentz, M., Scher, H., 2006. Modeling non-Fickian transport in geological formations as a continuous time random walk. Rev. Geophys. 44 (2), RG2003.

Bird, R.B., Stewart, W.E., Lightfoot, E.N., 1960. Transport Phenomena. Wiley \& Sons, New York. 780 pp.

Blümich, B., 2000. NMR Imaging of Materials. Clarendon Press, Oxford.

Boon, J.P., Yip, S., 1991. Molecular Hydrodynamics. Dover Publications, New York.

Brosten, T.R., Codd, S.L., Maier, R.S., Seymour, J.D., 2009. Dynamic length-scale characterization and nonequilibrium statistical mechanics of transport in open-cell foams. Phys. Rev. Lett. 103, 218001.

Callaghan, P.T., 1991. Principles of Nuclear Magnetic Resonance Microscopy. Oxford University Press, New York.

Callaghan, P.T., Codd, S.L., Seymour, J.D., 1999. Spatial coherence phenomena arising from translational spin motion in gradient spin echo experiments. Concepts Magn. Reson. 11, 181-202.

Clement, T.P., Hooker, B.S., Skeen, R.S., 1996. Macroscopic models for predicting changes in saturated porous media properties caused by microbial growth. Ground Water 34 (5), 934-942.

Cunningham, A.B., Gerlach, R., Spangler, L., Mitchell, A.C., 2009. Microbially enhanced geologic containment of sequestered supercritical $\mathrm{CO}_{2}$. Energy Procedia 1, 3245-3252.

Cushman, J.H., Ginn, T.R., 2000. Fractional advection dispersion equation: a classical mass balance with convolution-Fickian flux. Water Resour. Res. 36 (12), 3763-3766

Cushman, J.H., Hu, B.X., Ginn, T.R., 1994. Nonequilibrium statistical mechanics of preasymptotic dispersion. J. Stat. Phys. 75, 859-878.

Decho, A.W., 2010. Overview of biopolymer-induced mineralization: what goes on in biofilms? Ecol. Eng. 36, 137-144.

DeJong, J.T., Mortensen, B.M., Martinez, B.C., Nelson, D.C., 2010. Bio-mediated soil improvement. Ecol. Eng. 36, 197-2010.

Emmanuel, S., Berkowitz, B., 2005. Mixing-induced precipitation and porosity evolution in porous media. Adv. Water Resour. 28, 337-344. 
Emmanuel, S., Berkowitz, B., 2007. Effects of pore-size controlled solubility on reactive transport in heterogeneous rock. Geophys. Res. Lett. 34, L06404.

Ferris, F.G., Stehmeier, L.G., 1996. Bacteriogenic mineral plugging. Can. Pet. Technol. 35 (8), 56-61.

Ferris, F.G., Phoenix, V., Fujita, Y., Smith, R.W., 2003. Kinetics of calcite precipitation induced by ureolytic bacteria at 10 to $20{ }^{\circ} \mathrm{C}$ in artificial groundwater. Geochim. Cosmochim. Acta 67 (8), 1701-1722.

Graf von der Schulenberg, D.A., Akpa, B.S., Gladden, L.F., Johns, M.L., 2008. Non-invasive mass transfer measurements in complex biofilm-coated structures. Biotechnol. Bioeng. 101 (3), 602-608.

Hoskins, B.C., Fevang, L., Majors, P.D., Sharma, M.M., Georgiou, G., 1999. Selective imaging of biofilms in porous media by NMR relaxation. J. Magn. Reson. 139, 67-73.

Kaszuba, J.P., Janecky, D.R., Snow, M.G., 2005. Experimental evaluation of mixed fluid reactions between supercritical carbon dioxide and $\mathrm{NaCl}$ brine: relevance to the integrity of a geologic carbon repository. Chem. Geol. 217, 277-293.

Kersting, A.B., et al., 1999. Migration of plutonium in ground water at the Nevada test site. Nature 397, 56-59.

Koch, D.L., Brady, J.F., 1988. Anomalous diffusion in heterogeneous porous media. Phys. Fluids 31 (5), 965-973.

Kose, K., 1994. Spatial-mapping of velocity power spectra in Taylor-Couette flow using ultrafast NMR imaging. Phys. Rev. Lett. 72 (10), 1467-1470.

Lebon, L., et al., 1996. Pulsed gradient NMR measurements and numerical simulation of flow velocity distribution in sphere packings. Phys. Fluids 8 (2), 293-301.

Manz, B., Alexander, P., Gladden, L.F., 1999a. Correlations between dispersion and structure in porous media probed by nuclear magnetic resonance. Phys. Fluids 11 (2), 259-267.

Manz, B., Gladden, L.F., Warren, P.B., 1999b. Flow and dispersion in porous media: Lattice-Boltzmann and NMR studies. AlChE J. 45 (9), 1845-1854.
Mazo, R.M., 1967. Statistical Mechanical Theories of Transport Processes. The International Encyclopedia of Physical Chemistry and Chemical Physics, Topic 9:1. Pergamon Press, Oxford. 166 pp.

Nagy, B., et al., 1991. Organic matter and containment of uranium and fissiogenic isotopes at the Oklo natural reactors. Nature 354, 472-475.

Sahimi, M., Gavalas, G.R., Tsotsis, T.T., 1990. Statistical and continuum models of fluid-solid reactions in porous media. Chem. Eng. Sci. 45 (6), 1443-1502.

Salles, J., Thovert, J.-F., Delannay, L., Auriault, J.-L., Adler, P.M., 1993. Taylor dispersion in porous media. Phys. Fluids 5, 2348.

Scheven, U.M., Harris, R., Johns, M.L., 2007. Intrinsic dispersivity of randomly packed monodisperse spheres. Phys. Rev. Lett. 99, 054502.

Sederman, A.J., Mantle, M.D., Buckley, C., Gladden, L.F., 2004. MRI technique for measurement of velocity vectors, acceleration and autocorrelation functions in turbulent flow. J. Magn. Reson. 166 (2), 182-189.

Seymour, J.D., Callaghan, P.T., 1997. Generalized approach to NMR analysis of flow and dispersion in porous medium. AlChE J. 43, 2096-2111.

Seymour, J.D., Gage, J.P., Codd, S.L., Gerlach, R., 2004. Anomalous fluid transport in porous media induced by biofilm growth. Phys. Rev. Lett. 93 (19), 198103.

Seymour, J.D., Gage, J.P., Codd, S.L., Gerlach, R., 2007. Magnetic resonance microscopy of biofouling induced scale dependent transport in porous media. Adv. Water Resour. 30 (6-7), 1408-1420.

Tartakovsky, A.M., Meakin, P., Scheibe, T.D., Wood, B.D., 2007. A smoothed particle hydrodynamics model for reactive transport and mineral precipitation in porous and fractured porous media. Water Resour. Res. 43, W05437.

Thullner, M., Baveye, P., 2008. Computational pore network modeling of the influence of biofilm permeability on bioclogging in porous media. Biotechnol. Bioeng. 99 (6), 1337-1351. 